in vivo $35: 3449-3457(2021)$

doi:10.21873/invivo.12645

\title{
Embryonic Cell-free DNA in Spent Culture Medium: A Non-invasive Tool for Aneuploidy Screening of the Corresponding Embryos
}

\author{
AFRODITE SIALAKOUMA ${ }^{1,2}$, IOANNIS KARAKASILIOTIS ${ }^{3}$, VAIA NTALA ${ }^{2}$, \\ NIKOLAOS NIKOLETTOS ${ }^{4}$ and BYRON ASIMAKOPOULOS ${ }^{2}$ \\ ${ }^{1}$ IVF Assisted Reproduction Unit, Mitera General Hospital, Athens, Greece; \\ ${ }^{2}$ Laboratory of Physiology, Faculty of Medicine, Democritus University of Thrace, Alexandroupolis, Greece; \\ ${ }^{3}$ Laboratory of Biology, Faculty of Medicine, Democritus University of Thrace, Alexandroupolis, Greece; \\ ${ }^{4}$ Gynecological Clinic, General University Hospital of Alexandroupolis, \\ Democritus University of Thrace, Alexandroupolis, Greece
}

\begin{abstract}
Background/Aim: Preimplantation genetic testing (PGT) for chromosomal screening, based on embryo biopsy, has significant limitations. Cell-free DNA ( $c f-D N A)$ has been detected in spent culture medium (SCM), opening new horizons for the development of non-invasive PGT (ni-PGT). In this study, we evaluated the diagnostic performance of niPGT for aneuploidy (niPGT-A), comparing the results of trophectoderm biopsies (TE) and respective SCM from individually cultured embryos via Next Generation Sequencing (NGS). Materials and Methods: Forty fresh embryos were analyzed. TE and SCM from blastocysts were collected and analyzed. Results: We detected cfDNA in 100\% of samples tested. The overall concordance rate between the ni-PGT-A and PGT-A was 27/33 (81.8\%). The full concordance rate was $21 / 33$ (63.6\%). The aneuploidy agreement was $91.66 \%$, and the euploidy agreement was $76.19 \%$. Conclusion: We found a good accordance between TE and SCM analysis, suggesting that niPGT-A could be a reliable alternative for chromosomal abnormalities assessment of in vitro cultured embryos.
\end{abstract}

The development of in vitro fertilization (IVF) over the last four decades has reached significant milestones in infertility treatment. Nowadays, IVF is the most successful and

This article is freely accessible online.

Correspondence to: Afrodite Sialakouma, Mitera General Hospital, Assisted Reproduction Unit, 6, Erythrou Stavrou Str. 151 23, Marousi, Athens, Greece. Tel: +30 6978100540, e-mail: asialakouma@gmail.com

Key Words: cfDNA, culture medium, non-invasive PGT, trophectoderm biopsy. efficient approach to help infertile couples $(1,2)$. A key aspect in embryology, known since its introduction, is that different gametes have different potentials for successful growth, even in the most favorable environment. In the early days of IVF application, this knowledge led to the transfer of many embryos in each attempt, chosen by only morphological criteria $(3,4)$.

Multiple embryo transfers have an increased pregnancy rate, but this comes at a price of complications such as perinatal mortality, premature birth, etc. Across the world, various national legal frameworks have been established, stating that according to age, there is a restriction to how many embryos can be transferred to prevent multiple pregnancies and their complications. The international consensus is the selection and single transfer of the best possible healthy and viable embryo, which will lead to the birth of a living and healthy baby. One of the most critical dilemmas that all clinical embryologists have had is choosing the appropriate embryos for transfer.

Scientists are pursuing the goal of finding reliable embryo selection techniques and procedures. The different approaches to the best and most effective embryo selection technique or strategy can be divided into two broad categories, the non-invasive and the invasive. Invasive methods require the removal of specific cells from the embryo through biopsy to analyze its genetic composition.

Chromosomal makeup analysis of the embryo can serve as a prognostic factor of its implantation potential. Embryos with aneuploidy exhibit diminished potential of implanting and a lower pregnancy rate (5-8). The assessment of chromosomal status in each embryo can allow the selection of only euploid ones, significantly improving the results of in vitro fertilization (9). While this assumption has always been valid, the first attempts to examine fetal aneuploidy 
were insufficient $(10,11)$. At first, the molecular techniques that were applied were not accurate enough to be clinically relevant. More recently, molecular technology has constantly progressed in clinical sensitivity and specificity (12-15).

Preimplantation genetic testing (PGT) is an invasive procedure to acquire embryo DNA by removing some cells from the embryo through biopsy, but this has significant limitations. Embryo biopsy provides a "snapshot" in time of embryo DNA composition as embryo development occurs. Cells removal from day three embryos can dramatically affect their development $(16,17)$. Similarly, there is an inverse association between the number of cells derived from embryo biopsies and the likelihood of embryo implantation, especially in blastocysts with poor trophectoderm quality (18). Biopsies cannot be successfully performed in early blastocysts since there is a danger of accidental aspiration of the inner cell mass, as ICM lies very close to trophectoderm cells $(16,17)$. As a result, some healthy embryos with slower development are falsely deemed unsuitable candidates, thus resulting in the loss of potentially viable embryos.

Moreover, invasive procedures undermine the reproductive potential of embryos. Biopsy at the cleavage stage leads to impaired development of the fetus (19-22), with potential long-term adverse effects (23-25). Animal studies showed that offspring from biopsied embryos demonstrate epigenetic alterations and disorders in neurodegenerative tissues, adrenal glands, and ovarian deficiencies (26-30). Still, there are safety concerns about the prolonged culture of embryos up to the blastocyst stage to be accessible for trophectoderm biopsy $(31,32)$. Extensive embryo culture beyond the embryo's genome activation stage has been associated with an increased risk of monozygotism, perinatal mortality, genetic anomalies, premature birth, and infants $(33,34)$. Finally, invasive procedures cost more, are laborious, require more time, and demand highly skilled embryologists (17), so an alternative robust PGT method is needed.

Another aspect of embryo biopsies is that we can collect only a small portion of cells from specific points from the embryo, a limiting factor of genetic diagnosis of the embryo DNA integrity. As a result, we cannot identify mosaic embryos accurately because they show DNA heterogenicity, leading to a false genetic diagnosis (35-38). However, mosaic embryos can give birth to healthy babies $(39,40)$, but the dynamic implantation decreases compared to euploid embryos $(41,42)$. In addition, researchers have found a discrepancy between the chromosomal states of cells in trophectoderm (TE) biopsies, so these cells do not necessarily represent the whole embryo $(40,43,44)$.

Recent studies have shown detection of cf-DNA in biological fluids, blastocyst fluid, and spent culture medium of in vitro cultured embryos $(39,40,45-50)$, opening new roads for the implementation of non-invasive procedures in assisted reproductive technology. The cf-DNA that can be detected by the embryonic developmental culture material, SCM, seems to be the best choice for non-invasive PGT (niPGT). Many research groups have detected cfDNA and are under evaluation as a potential candidate method of assessing in vitro cultured embryo's chromosomal status $(26,39,40$, $45,48,51)$. A recent review of 15 published studies (46) showed that spent culture medium DNA detection is a safe and effective method for the chromosomal status of the developing embryos. However, the different methodologies followed in various studies undermine the validity of the findings as it is impossible to correlate the results directly.

Another problem is that several studies have detected extra-embryonic DNA in spend culture medium (SCM), a major limiting factor of the diagnostic and clinical performance of niPGT-A. Therefore, to implement niPGT-A procedures, we need to have concrete data derived from large-scale studies with comparable methodologies. Furthermore, provision must be taken to accurately determine the origin of the DNA detected (embryonic or non-embryonic).

In the present study, we compared the results of NGS aneuploidy control from TE biopsies and the SCM of the respective embryos in PGTA cycles. In addition, we tried to implement all the proposed measures to avoid contamination, to investigate the reliability of ni-PGT with optimized procedures. These results can offer helpful insight in research of reproductive biology.

\section{Patients and Methods}

Patients. We collected forty embryos from 13 infertile patients undergoing PGT-A in 2019-2020 at the IVF Unit of Mitera General Hospital in Athens.

Outcome assessments. The primary evaluation of this study was the embryos chromosomal status; for this reason, embryos were cultured to the blastocyst stage (d5/d6). NGS analyzed trophectoderm biopsies and respective SCMs from individually cultured embryos, and we compared the results. The average number of the collected eggs was $1.7+0.8$, from women with mean age 35.3+4.2. Before being biopsied and frozen, we assessed the blastocysts according to the Gardner system of classification (52).

Embryo culture - Embryo biopsy - Collection and storage of SCM. The oocyte's fertilization was performed by intracytoplasmic sperm injection (ICSI) in all cases, using only mature (metaphase II) oocytes. After ICSI, the fertilized oocytes were cultured in groups, up to 3 embryos per $25 \mu \mathrm{l}$ microdroplets, from day1 (D1) to day3 (D3), in Sage 1-Step culture material (Origio, Denmark) under oil (Origio).

We removed all remaining cumulus cells on day 3 of embryo culture and rinsed them thoroughly at least three times. Then we transferred to separate $10 \mu 1$ fresh microdroplets with Sage 1-Step culture material (Origio) under oil (Origio) for individual culture up to the blastocyst stage (day 5 or 6). Once the embryo has reached a fully expanded blastocyst, we collect and release the blastocyst fluid (BF) into the culture medium (SCM), then the blastocyst collapsed, 
and each blastocyst was hatched by laser in order some cells (3-5) to come out of the zona, those cells were collected for preimplantation genetic analysis. When the embryo was suitable for the biopsy procedure, we transferred them to a biopsy plate, taking the cells for genetic analysis. Then the embryo was cryopreserved by vitrification according to standard procedures (53). Finally, the combined SCM and BF samples, intended for non-invasive PGT-A, were collected and frozen at $-80^{\circ} \mathrm{C}$ until analyzed. Then, we performed total genome amplification (WGA) and analysis of these samples for niPGT-A.

Whole genomic amplification (WGA) and chromosome analysis. All trophectoderm samples after biopsies and cell-free DNA from blastocyst culture material were amplified using the SurePlex (BlueGnome Ltd., Mill Court, Great Shelford, Cambridge, UK) kit according to the manufacturing instructions and quantified by the Qubit 3.0 Fluorimeter (Thermo Fisher Scientific, Waltham, MA, USA). In addition, the amplified DNA was evaluated for complete and partial chromosome aneuploidy testing with a previously validated VeriSeq ${ }^{\mathrm{TM}}$ PGS kit in ThermoFisher ion Reproseq PGS 520 kit, for cell-free DNA and in Illumina VeriSeq PGS Kit with automatic analyzer Bluefuse multi-data analysis, which controls 24 chromosome aneuploidies for trophectoderm biopsies.

We set a reliability threshold for the results of Illumina's VeriSeq PGS Kit analysis system; when the DLR was $<0.4$, the read after filtering was $>150,000$, the average quality score was $>30$, and the average alignment score was $>30$. Regarding the ThermoFisher ion $\mathrm{R}$ proseq PGS 520 kit analysis system, we considered the results reliable when the MAPD was $<0.3$ the reads after filtering was $>100,000$

We used pure culture material as a negative control to check if there was cell-free DNA in material with no embryo development and to ensure the methodology of the technique.

Evaluation of chromosomal content. Both cf-DNA samples and TE biopsies were analyzed using the protocol as mentioned earlier. In addition, their chromosomal status (euploid $v s$. aneuploid) and the type and size of the chromosomal disorder in aneuploidy were evaluated using BlueFuse Multi software (Illumina, San Diego, CA, USA) and the ThermoFisher software.

We considered samples as full concordant when they were identical concerning the extent of polyploidy and mosaicism. Samples that agreed on the state of ploidy (euploid versus aneuploid) were considered as overall concordant, even if they differed in the type of aneuploidy.

We performed a chromosomal analysis of the whole embryo in specific samples deemed aneuploidy by TE biopsy and SCM. The patients permitted the destruction of the embryos. These samples are considered the gold standard in the process of evaluating the reliability of the niPGT-A method.

Statistical analysis. The PGT-A results by TE biopsies and niPGTA results by SCM were correlated and analyzed using the McNemar test; also, we performed ROC analysis. In all statistical tests, we set the level of statistical significance to $95 \%(p<0,05)$. In addition, we calculated $95 \%$ confidence intervals $(95 \% \mathrm{CI})$.

Ethical approval and consent to participants. The present study has permission from the Ethics committee of the Democritus University of Thrace and by the Council of Science of the Mitera Hospital in
Athens. Furthermore, all couples in this study have signed a consent agreement stating that free nucleic acids will be tested in the remaining culture material, otherwise rejected, by their in vitro cultured embryos as part of their assisted reproduction program. It is also explicitly stated that their decision to participate or not in this research would not affect the provision of services by the Assisted Reproduction Unit of Mother Hospital.

\section{Results}

In this study, we examined embryos with an unknown chromosomal profile. We performed trophectoderm biopsy on blastocysts of the fifth or sixth day of growth, i.e., PGT-A samples $(n=40)$. At the same time, the spent culture material (SCM) of the respective embryos was collected for the analysis of cell-free DNA, i.e., niPGT-A samples $(n=40)$ (Figure 1). A total of 40/40 samples were amplified after WGA and analyzed by NGS. We collected the SCM samples from embryos cultured for 48-72 h after day3 to amplify cfDNA. After wholegenome amplification (WGA), the DNA concentration in each sample ranged between 2,500-30,000 ng/ml for TE biopsies and 2,000-20,400 ng/ml for SCM. The negative controls (new and unused culture medium) showed overall no amplification. Although the culture time was different between the day 5 and day 6 blastocysts, the DNA concentration of the culture medium collected for niPGT-A was not statistically different concerning the day of collection (day5 embryo group: 20.0 $\mathrm{ng} / \mu \mathrm{l} v s$. day6 embryo group $20.8 \mathrm{ng} / \mu \mathrm{l} ; p=0.807$ ).

We categorized the samples as euploid, aneuploid, or mosaic, according to the results. Table I presents the analysis of the results. We excluded seven samples from the SCM due to the low quality of the DNA in the NGS analysis, so we included 33 samples in the PGT-A and niPGT-A analysis (Table II).

The concordance rate between autosomal and sex chromosomes of PGT-A and niPGT-A samples for each embryo was estimated (Table III). The full concordance rate between the ni-PGT-A and PGT-A from samples taken from the same embryo was $21 / 33$ (63.6\%). The overall concordance rate between the ni-PGT-A and PGT-A, from samples taken from the same embryo, was $27 / 33,81.8 \%$ (95\% CI=68-96\%). McNemar test: $p=0.687$ (non-statistically significant). The sex chromosomes concordance rate was $100 \%$.

To estimate the diagnostic accuracy of niPGT-A, we calculated sensitivity (i.e., true positive) and specificity (i.e., true negative) in the PGT-A and the niPGT-A (Table IV). The sensitivity (aneuploidy agreement) was $91.66 \%$, and the specificity (euploidy agreement) was $76.19 \%$. In ROC analysis, AUC was $82.3 \%(95 \% \mathrm{CI}=66.9-97.8)$. Of the 33 samples, 7 were male (XY), according to TE biopsy and SCM analysis, which confirms the safety of the method, as it shows no contamination by maternal DNA. In 5 samples, ni-PGTA from SCM detected euploidy, while TE biopsy showed embryomonosomes, possibly due to mosaicism. 


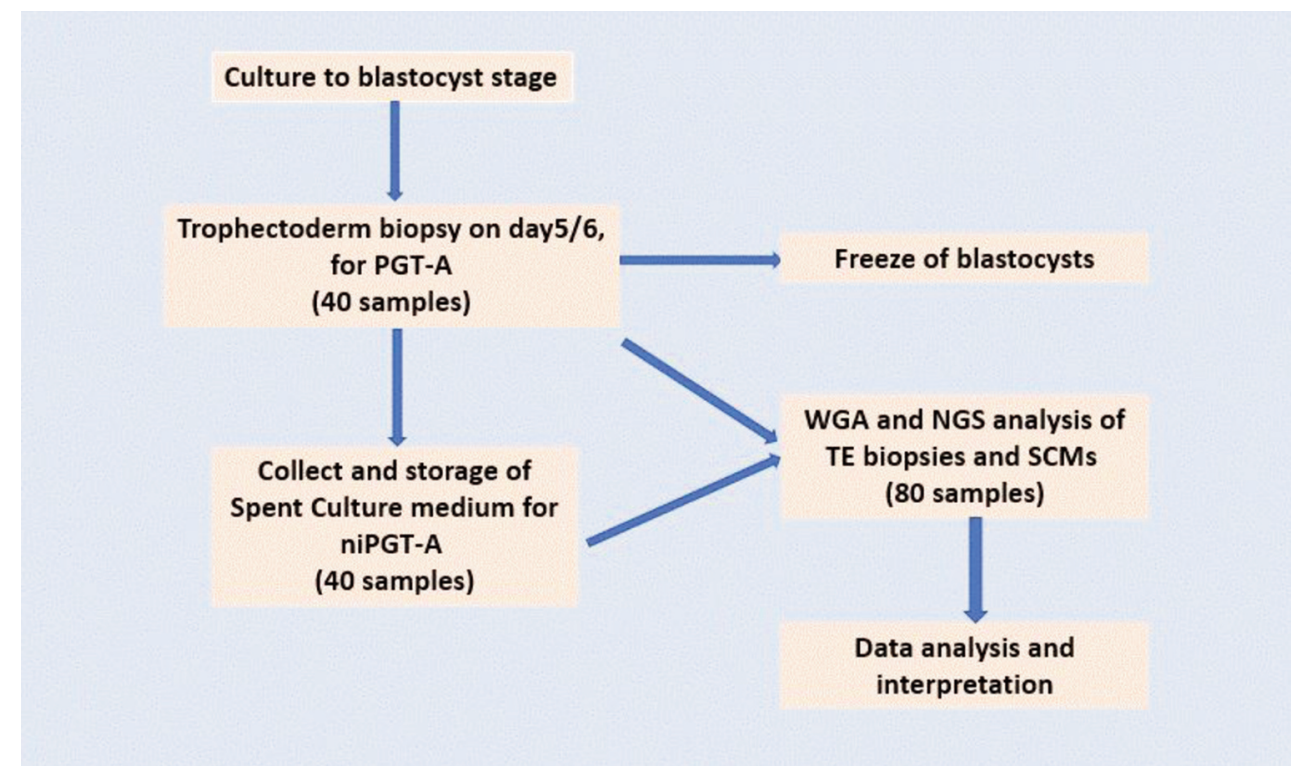

Figure 1. The study flow chart.

In 4 samples that have been detected aneuploid both by TE and SCM analysis, and we had permission from the patients, we performed a chromosomal analysis of the entire embryo, and we found aneuploidy as well (Table II; samples 14, 28, $33,34)$. In sample 33 , the results of the genetic analysis of the whole blastocyst were identical with the SCM analysis. Of particular interest is one sample, where trophectoderm biopsy showed euploidy, while niPGT showed a 6-fold increase in a small portion of chromosome 2 .

\section{Discussion}

Due to its limitations, PGT-A by TE biopsy cannot accurately depict the whole genome of the developing embryos. Several studies have documented PGT-A results through TE biopsy. The degree of congruity between the biopsies of trophectoderm and inner cell mass (ICM) karyotyping ranged between $62.1-86.2 \%(39,43,44,54)$. A recent study calculated that the sensitivity (probability of diagnosing an abnormality in ICM by TE biopsy) was $90.9 \%$, while the specificity (the percentage of embryos with normal ICM diagnosed as clinically appropriate), according to the TE biopsy, was $66.7 \%$ (44). Based on these findings, they estimated the overall diagnostic accuracy at $75 \%$. Furthermore, the overall rate of chromosomal mosaicism was determined in $37.9 \%$ of the examined embryos, suggesting that blastocysts are more chromosomaly diverse at a far more significant rate than reported before (44). These findings have shed doubts regarding the use of NGS, the PGT-A
Table I. Ploidy analysis of trophectoderm (TE) biopsied embryos and spend culture medium (SCM) of the corresponding embryos.

\begin{tabular}{lcc}
\hline & PGT-A & niPGT-A \\
\hline Number of analyzed embryos & 40 & 40 \\
Failed NGS analysis & $0(0 \%)$ & $7(17,5 \%)$ \\
Euploid & $16(40 \%)$ & $16(48,48 \%)$ \\
Aneuploid & $24(60 \%)$ & $17(51,5 \%)$ \\
Mosaic & $4(10 \%)$ & $0(0 \%)$ \\
\hline
\end{tabular}

NGS: Next generation sequencing; PGT-A: preimplantation genetic testing for aneuploidy; niPGT-A: non-invasive preimplantation genetic testing for aneuploidy.

diagnostic performance, and the predictive power of TE biopsy. In a recent study (40) in IVF cases where infertile couples had only blastocysts with aneuploidy or mosaicism after TE biopsy check, the patients decided to transfer the blastocyst with mosaicism with a clinical pregnancy rate of $50 \%$. Therefore, if a percentage of blastocyst mosaics can lead to healthy babies, the results of this procedure are undoubtedly at risk of their exclusion. However, the potential for embryo implantation and development with euploid ICM and mosaic or abnormal TE still needs to be elucidated. Currently, the whole blastocyst's DNA makeup is tough to precisely assess by TE biopsies $(39,40,44,54)$.

Biopsies of embryos have a documented negative impact on embryo dynamic and maybe undermine the implantation 
Table II. Chromosomal status of trophectoderm (TE) biopsies, spend culture media (SCM) and whole blastocysts. Data were obtained from 40 embryos from 13 patients.

\begin{tabular}{|c|c|c|c|c|c|c|}
\hline No & $\begin{array}{l}\text { Day of biopsy/ } \\
\text { freeze }\end{array}$ & Age & Grade & PGT-A & niPGT-A & $\begin{array}{l}\text { Whole } \\
\text { blastocyst }\end{array}$ \\
\hline 1 & 5 & 36 & 2BB & $46 X X$ & $46 X X$ & \\
\hline 2 & 5 & 36 & $4 \mathrm{AA}$ & $46 X X$ & N/A & \\
\hline 3 & 5 & 36 & $3 \mathrm{AA}$ & $46 X X$ & N/A & \\
\hline 4 & 5 & 38 & 3AA & $46 X Y$ & N/A & \\
\hline 5 & 5 & 38 & $3 \mathrm{AB}$ & $46 X X$ & $46 \mathrm{XX}$ & \\
\hline 6 & 5 & 38 & $3 \mathrm{AB}$ & $47 X X,+16,-14$ & $46 X X$ & \\
\hline 7 & 5 & 33 & $3 \mathrm{AA}$ & $46 X Y$ & N/A & \\
\hline 8 & 5 & 33 & $3 \mathrm{AA}$ & $46 X X$ & $46 X X$ & \\
\hline 9 & 5 & 33 & $3 \mathrm{AA}$ & $43 X X,-8,-10,-22$ & N/A & \\
\hline 10 & 5 & 36 & $4 \mathrm{AA}$ & 46XX & $46 X X$ & \\
\hline 11 & 5 & 36 & $4 \mathrm{AA}$ & $46 X X$ & $46 X X$ & \\
\hline 12 & 5 & 39 & $4 \mathrm{AA}$ & $44 X X,-15,-22$ & $45 X X,-15$ & \\
\hline 13 & 5 & 39 & $3 \mathrm{AA}$ & $46 \mathrm{XY},+3,-14(\mathrm{p} 21.2 ; \mathrm{q} 32.13)$ & $46 \mathrm{XY},+3,-14(\mathrm{p} 21.2 ; \mathrm{q} 32.13)$ & \\
\hline 14 & 6 & 40 & $5 \mathrm{AA}$ & $48 X X,+16,+22$ & $48 X X,+16,+22$ & $48 X X,+16,+22$ \\
\hline 15 & 6 & 40 & $5 \mathrm{AB}$ & $45 X Y,-16$ & N/A & \\
\hline 16 & 5 & 36 & $3 \mathrm{AB}$ & $46 X X$ & $46 X X$ & \\
\hline 17 & 6 & 36 & $4 \mathrm{AA}$ & $45 X X,-22$ & $45 X X,-22$ & \\
\hline 18 & 5 & 36 & $4 \mathrm{AA}$ & $47 \mathrm{XX},+15$ & N/A & \\
\hline 19 & 5 & 36 & $3 \mathrm{AA}$ & 46XX & $46 X X$ & \\
\hline 20 & 5 & 44 & $3 \mathrm{AA}$ & $45 X Y,+13,-19,-22$ & $4 X Y,+3,-6,-8,+13,-22$ & \\
\hline 21 & 5 & 44 & $3 \mathrm{AA}$ & $48 X Y,+18,+20,+22,-21$ & $46 X Y,+16,-21$ & \\
\hline 22 & 5 & 44 & $3 \mathrm{BB}$ & Chaotic, $\mathrm{XX}$ & Chaotic, XX & \\
\hline 23 & 5 & 44 & $4 \mathrm{AA}$ & $47 \mathrm{XY},+21$, mosaic & $46 \mathrm{XX}$ & \\
\hline 24 & 5 & 44 & $3 \mathrm{AB}$ & $43 X X,-10,-18,-20$ & $47 \mathrm{XX},+18$ & \\
\hline 25 & 5 & 44 & $4 \mathrm{AA}$ & $47 \mathrm{XX},+22$, mosaic & $46 X X$ & \\
\hline 26 & 5 & 39 & 3BB & $46 X X$ & $46 X X$ & \\
\hline 27 & 5 & 39 & $4 \mathrm{AA}$ & $46 X X$ & $46 X X$ & \\
\hline 28 & 5 & 39 & $3 \mathrm{AA}$ & $47 \mathrm{XX},+18$ & $47 \mathrm{XX},+18$ & $47 \mathrm{XX},+18$ \\
\hline 29 & 5 & 31 & $3 \mathrm{AA}$ & $46 X X$ & $46 X X$ & \\
\hline 30 & 5 & 31 & $3 \mathrm{AA}$ & $46 X X$ & $46 X X$ & \\
\hline 31 & 5 & 31 & $4 \mathrm{AA}$ & $47 \mathrm{XX},+13$, mosaic? & $46 X X$ & \\
\hline 32 & 5 & 31 & $3 \mathrm{AA}$ & $47 \mathrm{XX},+13$, mosaic? & $46 X X$ & \\
\hline 33 & 5 & 45 & $3 \mathrm{AB}$ & $45 X Y,-4,+7,-12,+20,-21$ & $47 X Y,-4,+7,+20$ & $47 X Y,-4,+7,+20$ \\
\hline 34 & 5 & 45 & $3 \mathrm{BB}$ & $46 X Y,+2,-13$ & $49 X Y,+2,-13$ & $49 X Y,+2,-13$ \\
\hline 35 & 6 & 41 & $4 \mathrm{AA}$ & $45 X X,-22$ & $45 X X,-22$ & \\
\hline 36 & 6 & 41 & $4 \mathrm{AA}$ & $48 X X,+9,+19$ & $47 X X,+9,+19$ & \\
\hline 37 & 6 & 41 & $5 \mathrm{AA}$ & $46 X X$ & $\begin{array}{l}46 \mathrm{XX}, 6 \text { times increase } \\
\text { in a small fragment of } 2 \text { chromosome }\end{array}$ & \\
\hline 38 & 6 & 41 & $5 \mathrm{AA}$ & $45 X X,-15$ & $45 \mathrm{XX},-15$ & \\
\hline 39 & 6 & 41 & $5 \mathrm{AA}$ & $48 X Y,+9,+18$ & $47 X Y,+9$ & \\
\hline 40 & 6 & 41 & $5 \mathrm{AA}$ & $46 X Y,-18,+19$ & $46 X Y,-18,+19$ & \\
\hline
\end{tabular}

PGT-A: Preimplantation genetic testing for aneuploidy; niPGT-A: non-invasive preimplantation genetic testing for aneuploidy.

potential $(21,39)$. Moreover, studies in animals have shown that embryo biopsy can cause neurodevelopment and adrenal disorders, but the impact in humans remains unclear $(26,29)$. A more reliable and efficient non-invasive method to produce clinically relevant results without the hazards of invasive methods would be a valuable tool for genetic screening in IVF clinics.

The cf-DNA detected by the embryonic developmental culture material is probably an excellent alternative to traditional invasive PGT. The niPGT-A methodology is easier than invasive methods and does not require expensive hardware, e.g., lasers. However, the diagnostic accuracy of niPGT-A is, for the moment, unclarified and under scrutiny. Previous researchers have reported a low, 20.8\%, concordance rate between TE and SCM for monosomy testing (39). In another study, the SCM/entire D3 embryo concordance rate was $56.3 \%$, increasing to $65 \%$ at d5 of the development (39). Jiao et al., using a modified MALABAC 
Table III. Chromosomal concordance rates of preimplantation genetic testing for aneuploidy (PGT-A) vs. non-invasive preimplantation genetic testing for aneuploidy (ni-PGT-A).

\begin{tabular}{lccc}
\hline & $\begin{array}{c}\text { Overall } \\
\text { concordance }\end{array}$ & $\begin{array}{c}\text { Full } \\
\text { concordance }\end{array}$ & $\begin{array}{c}\text { Sex } \\
\text { chromosomes } \\
\text { concordance }\end{array}$ \\
\hline PGT-A vs.niPGT-A & $27 / 33(81.8 \%)$ & $21 / 33(63.6 \%)$ & $33 / 33(100 \%)$ \\
\hline
\end{tabular}

method increased the agreement on chromosomal DNA between spent culture medium and blastocysts to $90 \%$ (39). In the present study, we estimated the agreement at the state of ploidy (overall concordance rate) between niPGT-A samples and PGT-A samples at $81.8 \%$.

We chose to collect the SCM two to three days after single embryo culture because in that time frame, as other studies have shown $(46,48)$, there is the highest amount possible of cfDNA with the lowest degradation rate. The quality and the quantity of cfDNA have a direct impact on the niPGT-A diagnostic accuracy. The rationale behind the blastocyst fluid and SCM mix is the qualitative and quantitative enhancement of cell-free DNA. Other research groups have also used this technique with good results (48). We can also confirm that this technique gives reasonable amounts of high-quality cfDNA. In our study, we found NGS applicable cfDNA in 33/40 samples. Although we had an amplification rate in all samples, the cfDNA has low quality in seven samples and thus did not give reliable results, probably due to degradation. We collect the SCM 48-72 h after culture, and maybe this time is extended for some DNA molecules, resulting in degradation. The secretory mechanism of cellfree DNA is unknown. A potential source would be from cells entering the apoptotic pathways inside the growing embryo, increasing the probability of degraded DNA (39, 48). The clinical performance of genetic testing is of paramount importance for preimplantation diagnosis; for that reason, we selected NGS, as it is a widely recognized methodology capable of providing timely and accurate results on chromosomal disorders and mosaic embryos (41).

We obtained a good correlation of PGT-A $v s$. niPGT-A results (overall concordance $81.8 \%, p=0.687$, nonstatistically significant). Maternal contamination DNA is a major limiting factor that can affect the diagnostic performance of the methodology. Previous studies have documented this effect $(48,51)$. In our study, we tried to optimize the whole procedure to avoid maternal or other contamination. We used Intracytoplasmic Sperm Injection (ICSI) as a fertilization procedure to avoid paternal contamination. We carefully denuded the oocytes from cumulus cells and rinsed them thoroughly carefully at least
Table IV. Aneuploidy/Euploidy agreement between preimplantation genetic testing for aneuploidy (PGT-A) vs. non-invasive preimplantation genetic testing for aneuploidy (ni-PGT-A) groups.

\begin{tabular}{lcc}
\hline & $\begin{array}{c}\text { Sensitivity } \\
\text { (aneuploidy } \\
\text { agreement) }\end{array}$ & $\begin{array}{c}\text { Specificity } \\
\text { (euploidy } \\
\text { agreement) }\end{array}$ \\
\hline PGT-A vs. niPGT-A & $91.66 \%$ & $76.19 \%$ \\
\hline
\end{tabular}

three times in fresh medium microdroplets. The embryos after day 3 were cultured in single microdroplets up to the blastocyst stage. We had seven male samples (XY) and twenty-six females (XX); both TE biopsy and SCM analysis confirm these results, suggesting that this methodology is robust and safe. A further step to ensure the diagnostic accuracy of this methodology could be the analysis of the XX samples for single nucleotide polymorphisms (SNPs) to establish the origin of DNA, maternal or embryonic.

We found a discrepancy in euploidy status in 4 samples (Table II, samples 23, 25, 31, 32). TE biopsy showed aneuploidy, but SCM showed euploidy. We classified those embryos as mosaics. Other studies have also found various degrees of discrepancies (48). Li et al. (40) reported that when mosaic embryos were re-cultured and re-tested the whole blastocyst, they found euploidy at $85.4 \%$. They also noted that setting the threshold of mosaicism at 50\%, the niPGT-A concordance rate between the SCM and Whole Blastocyst analysis was $87.2 \%$, more significant than the concordance rate with TE biopsy, suggesting that niPGT-A can more accurately diagnose the ploidy status of mosaic embryos. We collected DNA from both the blastocyst fluid and the spent culture medium, and we believe that this approach gives more representative results of the ICM chromosome status (48).

Interestingly, in sample 13, a translocation [t $(3: 14)$ (p21.2;q32.13)] of paternal origin was detected, both TE biopsy and SCM, showing the diagnostic power of niPGT-A in detecting various types of chromosomal abnormalities. Of particular interest is 1 sample, where trophectoderm biopsy showed euploidy, while niPGT-A showed a 6-fold increase in a small portion of chromosome 2; it is possible that this increase in such a small part of the chromosome cannot be detected in trophectoderm cells, which may not be representative of the whole embryo, while cfDNA may be more representative.

Whether the cfDNA in the SCM represents the full chromosomal status of the embryo remains to be clarified. However, a blastocyst expansion study using DNA-specific fluorochromes revealed that the source of apoptotic cells is mainly from the inner cell mass (54). Indeed, if the origin of this DNA is predominantly from the inner cell mass, niPGTA may give a more reliable picture of the future fetus (47). 
Of the four samples we analyzed of the entire embryo, we found complete agreement between the TE biopsy, SCM, and Whole Blastocyst (WB) analysis in 3 out of the 4. In sample 33 , we found a $100 \%$ match with the study of the cfDNA from the SCM. Thus, the WB analysis is a robust diagnostic performance index of the niPGT-A methodology, even if the samples are few.

Time-dependent degradation of DNA and contamination of maternal DNA are risks that must be accounted for (48). However, the careful removal of cumulus cells and the meticulous washing of the embryos in single culture microdroplets can significantly reduce the risk of maternal DNA contamination. At the same time, using ICSI as a fertilization method can help avoid the risk of paternal DNA contamination.

\section{Conclusion}

Genetic analysis in IVF settings is widely used to select viable embryos, leading to healthy offsprings; however, there is a need to replace the invasive procedure with safer and non invasive techniques. We used niPGT-A to assess the chromosomal status of embryos. Our results showed that cfDNA from SCM could be detected and amplified at $100 \%$, but at a rate of $82.5 \%$, the cfDNA samples were appropriate for analysis. This rate can be even more increased through further refinement of the collection and amplification steps. In this study, the small number of samples is a limitation; nonetheless, we managed to fully determine the genetic makeup of specific blastocyst used for this research, a gold standard for the accurate determination of this methodology. Despite the limitations, we can confirm that niPGT-A, a non-invasive process with minimal risks to embryos, is reliable and precise and can be used along invasive PGTA as an alternative, especially in cases of mosaicism. Large-scale randomized control studies will be able to validate this very promising methodology and establish niPGT-A as a valuable tool to assist infertile couples in having healthy babies.

\section{Conflicts of Interest}

There are no conflicts of interest.

\section{Authors' Contributions}

Afrodite Sialakouma conceived the study, recruited the patients, performed the biopsies, collected the samples, performed the experiments, analyzed the data, wrote and edited the manuscript. Ioannis Karakasiliotis performed the experiments, analyzed the data, revised the manuscript. Vaia Ntala analyzed the data and revised the manuscript. Nikolaos Nikolettos and Byron Asimakopoulos revised the manuscript.

\section{Acknowledgments}

This study was funded in the context of the project "Investigation on the use of free nucleic acids in embryonic culture medium as a noninvasive tool for preimplantation genetic diagnosis" (MIS 5050321). The project is co-financed by Greece and the European Union (European Social Fund) by the Operation Program Human Recourses Development, Education and Lifelong Learning 2014-2020.

\section{References}

1 Goldman MB, Thornton KL, Ryley D, Alper MM, Fung JL, Hornstein MD and Reindollar RH: A randomized clinical trial to determine optimal infertility treatment in older couples: the Forty and Over Treatment Trial (FORT-T). Fertil Steril 101(6): 1574-81.e1-2, 2014. PMID: 24796764. DOI: 10.1016/j.fertnstert. 2014.03.012

2 Reindollar RH, Regan MM, Neumann PJ, Levine BS, Thornton $\mathrm{KL}$, Alper MM and Goldman MB: A randomized clinical trial to evaluate optimal treatment for unexplained infertility: the fast track and standard treatment (FASTT) trial. Fertil Steril 94(3): 888-899, 2010. PMID: 19531445. DOI: 10.1016/j.fertnstert.2009.04.022

3 Fishel SB, Edwards RG and Evans CJ: Human chorionic gonadotropin secreted by preimplantation embryos cultured in vitro. Science 223(4638): 816-818, 1984. PMID: 6546453. DOI: $10.1126 /$ science. 6546453

4 Fishel SB, Edwards RG and Walters DE: Follicular steroids as a prognosticator of successful fertilization of human oocytes in vitro. J Endocrinol 99(2): 335-344, 1983. PMID: 6686245. DOI: $10.1677 /$ joe. 0.0990335

5 Fragouli E and Wells D: Aneuploidy in the human blastocyst. Cytogenet Genome Res 133(2-4): 149-159, 2011. PMID: 21252488. DOI: $10.1159 / 000323500$

6 Gat I, Tang K, Quach K, Kuznyetsov V, Antes R, Filice M, Zohni K and Librach C: Sperm DNA fragmentation index does not correlate with blastocyst aneuploidy or morphological grading. PLoS One 12(6): e0179002, 2017. PMID: 28591199. DOI: $10.1371 /$ journal.pone.0179002

7 Kort JD, McCoy RC, Demko Z and Lathi RB: Are blastocyst aneuploidy rates different between fertile and infertile populations? J Assist Reprod Genet 35(3): 403-408, 2018. PMID: 29063503. DOI: 10.1007/s10815-017-1060-x

8 Gruhn JR, Zielinska AP, Shukla V, Blanshard R, Capalbo A, Cimadomo D, Nikiforov D, Chan AC, Newnham LJ, Vogel I, Scarica C, Krapchev M, Taylor D, Kristensen SG, Cheng J, Ernst E, Bjørn AB, Colmorn LB, Blayney M, Elder K, Liss J, Hartshorne G, Grøndahl ML, Rienzi L, Ubaldi F, McCoy R, Lukaszuk K, Andersen CY, Schuh M and Hoffmann ER: Chromosome errors in human eggs shape natural fertility over reproductive life span. Science 365(6460): 1466-1469, 2019. PMID: 31604276. DOI: 10.1126/science.aav7321

9 Liñán A, Lawrenz B, El Khatib I, Bayram A, Arnanz A, Rubio C, Chopra R and Fatemi HM: Clinical reassessment of human embryo ploidy status between cleavage and blastocyst stage by Next Generation Sequencing. PLoS One 13(8): e0201652, 2018. PMID: 30133476. DOI: 10.1371/journal.pone.0201652

10 Fritz MA: Perspectives on the efficacy and indications for preimplantation genetic screening: where are we now? Hum Reprod 23(12): 2617-2621, 2008. PMID: 19015133. DOI: 10.1093/humrep/den400 
11 Mastenbroek S, Twisk M, van Echten-Arends J, SikkemaRaddatz B, Korevaar JC, Verhoeve HR, Vogel NE, Arts EG, de Vries JW, Bossuyt PM, Buys CH, Heineman MJ, Repping S and van der Veen F: In vitro fertilization with preimplantation genetic screening. N Engl J Med 357(1): 9-17, 2007. PMID: 17611204. DOI: 10.1056/NEJMoa067744

12 Bouvier S, Paulmyer-Lacroix O, Molinari N, Bertaud A, Paci M, Leroyer A, Robert S, Dignat George F, Blot-Chabaud M and Bardin N: Soluble CD146, an innovative and non-invasive biomarker of embryo selection for in vitro fertilization. PLoS One 12(3): e0173724, 2017. PMID: 28291830. DOI: 10.1371/ journal.pone. 0173724

13 Munné S, Kaplan B, Frattarelli JL, Child T, Nakhuda G, Shamma FN, Silverberg K, Kalista T, Handyside AH, Katz-Jaffe M, Wells D, Gordon T, Stock-Myer S, Willman S and STAR Study Group: Preimplantation genetic testing for aneuploidy versus morphology as selection criteria for single frozen-thawed embryo transfer in good-prognosis patients: a multicenter randomized clinical trial. Fertil Steril 112(6): 1071-1079.e7, 2019. PMID: 31551155 . DOI: 10.1016/j.fertnstert.2019.07.1346

14 Rubio C, Bellver J, Rodrigo L, Castillón G, Guillén A, Vidal C, Giles J, Ferrando M, Cabanillas S, Remohí J, Pellicer A and Simón C: In vitro fertilization with preimplantation genetic diagnosis for aneuploidies in advanced maternal age: a randomized, controlled study. Fertil Steril 107(5): 1122-1129, 2017. PMID: 28433371. DOI: 10.1016/j.fertnstert.2017.03.011

15 Ou Z, Chen Z, Yin M, Deng Y, Liang Y, Wang W, Yao Y and Sun L: Re-analysis of whole blastocysts after trophectoderm biopsy indicated chromosome aneuploidy. Hum Genomics 14(1): 3, 2020. PMID: 31931889. DOI: 10.1186/s40246-0190253-z

16 Harton G, Braude P, Lashwood A, Schmutzler A, TraegerSynodinos J, Wilton L, Harper JC and European Society for Human Reproduction and Embryology (ESHRE) PGD Consortium: ESHRE PGD consortium best practice guidelines for organization of a PGD centre for PGD/preimplantation genetic screening. Hum Reprod 26(1): 14-24, 2011. PMID: 20966460. DOI: 10.1093/humrep/deq229

17 Milachich T: New advances of preimplantation and prenatal genetic screening and noninvasive testing as a potential predictor of health status of babies. Biomed Res Int 2014: 306505, 2014. PMID: 24783200. DOI: 10.1155/2014/306505

18 Zhang S, Luo K, Cheng D, Tan Y, Lu C, He H, Gu Y, Lu G, Gong F and Lin G: Number of biopsied trophectoderm cells is likely to affect the implantation potential of blastocysts with poor trophectoderm quality. Fertil Steril 105(5): 1222-1227.e4, 2016. PMID: 26820770. DOI: 10.1016/j.fertnstert.2016.01.011

19 Kirkegaard K, Hindkjaer JJ and Ingerslev HJ: Human embryonic development after blastomere removal: a time-lapse analysis. Hum Reprod 27(1): 97-105, 2012. PMID: 22081251. DOI: 10.1093/humrep/der382

20 Munné S, Gianaroli L, Tur-Kaspa I, Magli C, Sandalinas M, Grifo J, Cram D, Kahraman S, Verlinsky Y and Simpson JL: Substandard application of preimplantation genetic screening may interfere with its clinical success. Fertil Steril 88(4): 781-784, 2007. PMID: 17920402. DOI: $10.1016 /$ j.fertnstert.2007.08.002

21 Scott KL, Hong KH and Scott RT Jr: Selecting the optimal time to perform biopsy for preimplantation genetic testing. Fertil Steril 100(3): 608-614, 2013. PMID: 23993663. DOI: 10.1016/ j.fertnstert.2013.07.004
22 Scott RT Jr, Upham KM, Forman EJ, Zhao T and Treff NR: Cleavage-stage biopsy significantly impairs human embryonic implantation potential while blastocyst biopsy does not: a randomized and paired clinical trial. Fertil Steril 100(3): 624-630, 2013. PMID: 23773313. DOI: 10.1016/j.fertnstert.2013.04.039

23 Bay B, Ingerslev HJ, Lemmen JG, Degn B, Rasmussen IA and Kesmodel US: Preimplantation genetic diagnosis: a national multicenter obstetric and neonatal follow-up study. Fertil Steril 106(6): 1363-1369.e1, 2016. PMID: 27542705. DOI: 10.1016/ j.fertnstert.2016.07.1092

24 Middelburg KJ, Heineman MJ, Haadsma ML, Bos AF, Kok JH and Hadders-Algra M: Neurological condition of infants born after in vitro fertilization with preimplantation genetic screening. Pediatr Res 67(4): 430-434, 2010. PMID: 20057341. DOI: 10.1203/PDR.0b013e3181d2273e

25 Middelburg KJ, van der Heide M, Houtzager B, JongbloedPereboom M, Fidler V, Bos AF, Kok J, Hadders-Algra M and PGS Follow-up Study Group: Mental, psychomotor, neurologic, and behavioral outcomes of 2-year-old children born after preimplantation genetic screening: follow-up of a randomized controlled trial. Fertil Steril 96(1): 165-169, 2011. PMID: 21616485. DOI: 10.1016/j.fertnstert.2011.04.081

$26 \mathrm{Wu}$ Y, Lv Z, Yang Y, Dong G, Yu Y, Cui Y, Tong M, Wang L, Zhou Z, Zhu H, Zhou Q and Sha J: Blastomere biopsy influences epigenetic reprogramming during early embryo development, which impacts neural development and function in resulting mice. Cell Mol Life Sci 71(9): 1761-1774, 2014. PMID: 24037382. DOI: 10.1007/s00018-013-1466-2

27 Yu Y, Wu J, Fan Y, Lv Z, Guo X, Zhao C, Zhou R, Zhang Z, Wang F, Xiao M, Chen L, Zhu H, Chen W, Lin M, Liu J, Zhou Z, Wang L, Huo R, Zhou Q and Sha J: Evaluation of blastomere biopsy using a mouse model indicates the potential high risk of neurodegenerative disorders in the offspring. Mol Cell Proteomics 8(7): 1490-1500, 2009. PMID: 19279043. DOI: 10.1074/mcp.M800273-MCP200

28 Yu Y, Zhao Y, Li R, Li L, Zhao H, Li M, Sha J, Zhou Q and Qiao J: Assessment of the risk of blastomere biopsy during preimplantation genetic diagnosis in a mouse model: reducing female ovary function with an increase in age by proteomics method. J Proteome Res 12(12): 5475-5486, 2013. PMID: 24156634. DOI: $10.1021 / \mathrm{pr} 400366 \mathrm{j}$

29 Zeng Y, Lv Z, Gu L, Wang L, Zhou Z, Zhu H, Zhou Q and Sha $\mathrm{J}$ : Preimplantation genetic diagnosis (PGD) influences adrenal development and response to cold stress in resulting mice. Cell Tissue Res 354(3): 729-741, 2013. PMID: 24104561. DOI: 10.1007/s00441-013-1728-1

30 Zhao HC, Zhao Y, Li M, Yan J, Li L, Li R, Liu P, Yu Y and Qiao $\mathrm{J}$ : Aberrant epigenetic modification in murine brain tissues of offspring from preimplantation genetic diagnosis blastomere biopsies. Biol Reprod 89(5): 117, 2013. PMID: 24089199. DOI: 10.1095/biolreprod.113.109926

31 Braakhekke M, Kamphuis EI, Mol F, Norman RJ, Bhattacharya S, van der Veen $\mathrm{F}$ and Mol BW: Effectiveness and safety as outcome measures in reproductive medicine. Hum Reprod 30(10): 22492251, 2015. PMID: 26384402. DOI: 10.1093/humrep/dev201

32 Mani S and Mainigi M: Embryo culture conditions and the epigenome. Semin Reprod Med 36(3-04): 211-220, 2018. PMID: 30866008. DOI: $10.1055 / \mathrm{s}-0038-1675777$

33 Dar S, Lazer T, Shah PS and Librach CL: Neonatal outcomes among singleton births after blastocyst versus cleavage stage 
embryo transfer: a systematic review and meta-analysis. Hum Reprod Update 20(3): 439-448, 2014. PMID: 24480786. DOI: 10.1093/humupd/dmu001

34 Martins WP, Nastri CO, Rienzi L, van der Poel SZ, Gracia C and Racowsky C: Blastocyst vs. cleavage-stage embryo transfer: systematic review and meta-analysis of reproductive outcomes. Ultrasound Obstet Gynecol 49(5): 583-591, 2017. PMID: 27731533. DOI: $10.1002 /$ uog. 17327

35 McCoy RC: Mosaicism in preimplantation human embryos: when chromosomal abnormalities are the norm. Trends Genet 33(7): 448-463, 2017. PMID: 28457629. DOI: 10.1016/j.tig. 2017.04.001

36 Sachdev NM, Maxwell SM, Besser AG and Grifo JA: Diagnosis and clinical management of embryonic mosaicism. Fertil Steril 107(1): 6-11, 2017. PMID: 27842993. DOI: 10.1016/j.fertnstert. 2016.10.006

37 Taylor TH, Gitlin SA, Patrick JL, Crain JL, Wilson JM and Griffin DK: The origin, mechanisms, incidence and clinical consequences of chromosomal mosaicism in humans. Hum Reprod Update 20(4): 571-581, 2014. PMID: 24667481. DOI: 10.1093/humupd/dmu016

38 Vera-Rodriguez $\mathrm{M}$ and Rubio C: Assessing the true incidence of mosaicism in preimplantation embryos. Fertil Steril 107(5): 1107-1112, 2017. PMID: 28433370. DOI: 10.1016/j.fertnstert. 2017.03.019

39 Shitara A, Takahashi K, Goto M, Takahashi H, Iwasawa T, Onodera Y, Makino K, Miura H, Shirasawa H, Sato W, Kumazawa Y and Terada Y: Cell-free DNA in spent culture medium effectively reflects the chromosomal status of embryos following culturing beyond implantation compared to trophectoderm biopsy. PLoS One 16(2): e0246438, 2021. PMID: 33571233. DOI: 10.1371/journal.pone.0246438

40 Li X, Hao Y, Chen D, Ji D, Zhu W, Zhu X, Wei Z, Cao Y, Zhang Z and Zhou P: Non-invasive preimplantation genetic testing for putative mosaic blastocysts: a pilot study. Hum Reprod 36(7): 20202034, 2021. PMID: 33974705. DOI: 10.1093/humrep/deab080

41 Maxwell SM and Grifo JA: Should every embryo undergo preimplantation genetic testing for aneuploidy? A review of the modern approach to in vitro fertilization. Best Pract Res Clin Obstet Gynaecol 53: 38-47, 2018. PMID: 30146380. DOI: 10.1016/j.bpobgyn.2018.07.005

42 Munné S, Blazek J, Large M, Martinez-Ortiz PA, Nisson H, Liu E, Tarozzi N, Borini A, Becker A, Zhang J, Maxwell S, Grifo J, Babariya D, Wells D and Fragouli E: Detailed investigation into the cytogenetic constitution and pregnancy outcome of replacing mosaic blastocysts detected with the use of high-resolution nextgeneration sequencing. Fertil Steril 108(1): 62-71.e8, 2017. PMID: 28579407. DOI: 10.1016/j.fertnstert.2017.05.002

43 Popovic M, Dhaenens L, Taelman J, Dheedene A, Bialecka M, De Sutter P, Chuva de Sousa Lopes SM, Menten B and Heindryckx $\mathrm{B}$ : Extended in vitro culture of human embryos demonstrates the complex nature of diagnosing chromosomal mosaicism from a single trophectoderm biopsy. Hum Reprod 34(4): 758-769, 2019. PMID: 30838420. DOI: 10.1093/humrep/dez012

44 Popovic M, Dheedene A, Christodoulou C, Taelman J, Dhaenens L, Van Nieuwerburgh F, Deforce D, Van den Abbeel E, De Sutter P, Menten B and Heindryckx B: Chromosomal mosaicism in human blastocysts: the ultimate challenge of preimplantation genetic testing? Hum Reprod 33(7): 1342-1354, 2018. PMID: 29796631. DOI: 10.1093/humrep/dey106
45 Assou S, Aït-Ahmed O, El Messaoudi S, Thierry AR and Hamamah S: Non-invasive pre-implantation genetic diagnosis of X-linked disorders. Med Hypotheses 83(4): 506-508, 2014. PMID: 25182520. DOI: 10.1016/j.mehy.2014.08.019

46 Brouillet S, Martinez G, Coutton C and Hamamah S: Is cell-free DNA in spent embryo culture medium an alternative to embryo biopsy for preimplantation genetic testing? A systematic review. Reprod Biomed Online 40(6): 779-796, 2020. PMID: 32417199. DOI: $10.1016 /$ j.rbmo.2020.02.002

47 Handyside AH: Noninvasive preimplantation genetic testing: dream or reality? Fertil Steril 106(6): 1324-1325, 2016. PMID: 27645293. DOI: $10.1016 /$ j.fertnstert.2016.08.046

48 Kuznyetsov V, Madjunkova S, Antes R, Abramov R, Motamedi G, Ibarrientos Z and Librach C: Evaluation of a novel noninvasive preimplantation genetic screening approach. PLoS One 13(5): e0197262, 2018. PMID: 29746572. DOI: 10.1371/ journal.pone. 0197262

49 Lo YM, Corbetta N, Chamberlain PF, Rai V, Sargent IL, Redman $\mathrm{CW}$ and Wainscoat JS: Presence of fetal DNA in maternal plasma and serum. Lancet 350(9076): 485-487, 1997. PMID: 9274585. DOI: 10.1016/S0140-6736(97)02174-0

50 Stigliani S, Anserini P, Venturini PL and Scaruffi P: Mitochondrial DNA content in embryo culture medium is significantly associated with human embryo fragmentation. Hum Reprod 28(10): 2652-2660, 2013. PMID: 23887072. DOI: 10.1093/humrep/det314

51 Vera-Rodriguez M, Diez-Juan A, Jimenez-Almazan J, Martinez S, Navarro R, Peinado V, Mercader A, Meseguer M, Blesa D, Moreno I, Valbuena D, Rubio C and Simon C: Origin and composition of cell-free DNA in spent medium from human embryo culture during preimplantation development. Hum Reprod 33(4): 745-756, 2018. PMID: 29471395. DOI: 10.1093/ humrep/dey028

52 Gardner DK and Schoolcraft WB: In vitro culture of human blastocysts. Towar Reprod Certain Fertil Genet beyond. London, The Parthenon Publishing Group, 1999.

53 Kovačič B, Taborin M and Vlaisavljević V: Artificial blastocoel collapse of human blastocysts before vitrification and its effect on re-expansion after warming - a prospective observational study using time-lapse microscopy. Reprod Biomed Online 36(2): 121-129, 2018. PMID: 29212605. DOI: 10.1016/ j.rbmo.2017.10.111

54 Hammond ER, Shelling AN and Cree LM: Nuclear and mitochondrial DNA in blastocoele fluid and embryo culture medium: evidence and potential clinical use. Hum Reprod 31(8): 1653-1661, 2016. PMID: 27270971. DOI: 10.1093/humrep/ dew 132

55 Lawrenz B, El Khatib I, Liñán A, Bayram A, Arnanz A, Chopra R, De Munck N and Fatemi HM: The clinicians' dilemma with mosaicism-an insight from inner cell mass biopsies. Hum Reprod 34(6): 998-1010, 2019. PMID: 31114858. DOI: 10.1093/ humrep/dez055
Received July 26, 2021

Revised August 29, 2021

Accepted September 16, 2021 